\title{
Comparative studies of types 1 and 2 herpes simplex virus infection of cultured normal keratinocytes
}

\author{
S J Su, H H Wu, Y H Lin, H Y Lin
}

\begin{abstract}
Aims-To investigate the differences in biological properties, multiplication patterns, and cytopathic effects between type 1 and type 2 herpes simplex virus (HSV) through the replication of $\mathrm{HSV}$ in cultured normal human keratinocytes.

Methods-Keratinocytes were obtained from surgical specimens of normal gingiva, cervix, trunk skin, and newborn foreskin. They were cultured in serum free, chemically defined, culture medium and infected with a pool of HSV collected from clinical specimens.

Results-The reproductive patterns of HSV type 1 (HSV-1) and HSV type 2 (HSV-2) differed from each other regardless of the anatomical source of the cultured cells. This was made evident by the dissimilarity of their growth curves and cytopathic effects. The growth curve of HSV-2 showed a more or less continuously rising titre, whereas HSV-1 titres varied substantially at different time intervals. The cytopathic effects induced by HSV-1 infection took 24 more incubation hours than those induced by HSV-2 infection to manifest. During the early stages, the cytopathic changes of the two viruses looked different. However, all cultured cells, whether cultured with HSV-1 or HSV-2, eventually became small and globular in shape. The infective titres of both HSV-1 and HSV-2 were higher in infected cultured cervix than in infected cultured normal gingiva.

Conclusions-These data suggest that each serotype of HSV has its own unique replication pattern in human keratinocytes regardless of the cell origin.

(f Clin Pathol 1995;48:75-79)
\end{abstract}

Keywords: Herpes simplex virus, normal human keratinocytes.

Department of

Pathology, Taipei

Medical College

Hospital

S J Su

Y H Lin

H Y L in

School of Medical Technology, Taipei Medical College H H Wu

Correspondence to: Dr S J Su, Department of Pathology, Taipei Medical Street, Taipei 105, Taiwan.

Accepted for publication 10 May 1994 The differences in the various biological properties between herpes simplex virus type 1 (HSV-1) and herpes simplex virus type 2 (HSV-2) have been investigated before. However, the host cells in vivo and the cells used in in vitro studies are not quite the same. Some results obtained from previous in vitro studies have not correlated well with the clinical manifestations produced by the viruses.

Clinically, HSV-1 is often associated with the genital region, although recently it has been recognised that both type 1 and type 2 viruses can be present in any part of the body. Oral-labial HSV-1 infections recur more frequently than oral-labial HSV-2 infections, ${ }^{1}$ and it has also been reported that genital infections caused by HSV-1 are usually less severe and seem to recur less frequently than those caused by HSV-2. ${ }^{23}$ Lafferty et $a l^{4}$ found that there were differences in the subsequent recurrence pattern of $\mathrm{HSV}$ infections affecting the same anatomical regions between type 1 and type 2, and also between the oral-labial and genital areas infected either by type 1 or by type 2 virus. The clinical recurrence rate of genital HSV infection was six times greater than that of oral-labial HSV infection. Variability among viral types was also noticed, with genital recurrence developing in $89 \%$ of patients with HSV-2 infection compared with $25 \%$ of patients with HSV-1 infection. These clinical findings correlate with the previous results of in vitro studies reported by Birch et $a l .{ }^{5}$ Using organ culture of endocervical tissue, they found that HSV-2 replicated with higher titres than HSV-1. Conversely, Plummer et al demonstrated that the titres of viral infectivity harvested from cultured rabbit kidney cells infected with HSV-2 and from conditioned media were much lower than those infected with HSV-1.

The distinction between the virus types is relatively straightforward, clinically, but examination of cellular samples obtained from clinically infected patients shows that the cytopathic effects produced by HSV-1 and HSV-2 cannot be differentiated morphologically. However, animal cells, cultured human fibroblasts, and other cell lines infected with these two types of HSV show distinct cytopathic manifestations and vary in the rate of development. ${ }^{67}$ However, no clinically important difference in induction of cytopathic effects was observed in cultured human term trophoblasts. ${ }^{8}$

Most previous in vitro studies have been oral infection, while HSV-2 is mostly found in performed on organ cultures, animal cells, and human fibroblasts as well as on cancer cell lines. This might be one of the causes of the discrepancies between the clinical findings and the results of in vitro studies on viral biological behaviour. Taking into account the advantages of recent advances in the culture of human keratinocytes, we cultured normal keratinocytes from various human anatomical sites, which form the host cells for clinical infection with HSV. 
Figure 1 Growth curves of intracellular HSV-1 and $H S V-2$ in infected cervix and normal gingiva. Each data point represents mean experiments (bar not drawn when titre difference between duplicate is les than $0.08 \log \mathrm{pfu} / \mathrm{ml}$ ). C: cervix; $G$ : normal gingiva; 1: $H S V-1 ; 2: H S V-2$. (range) of duplicate

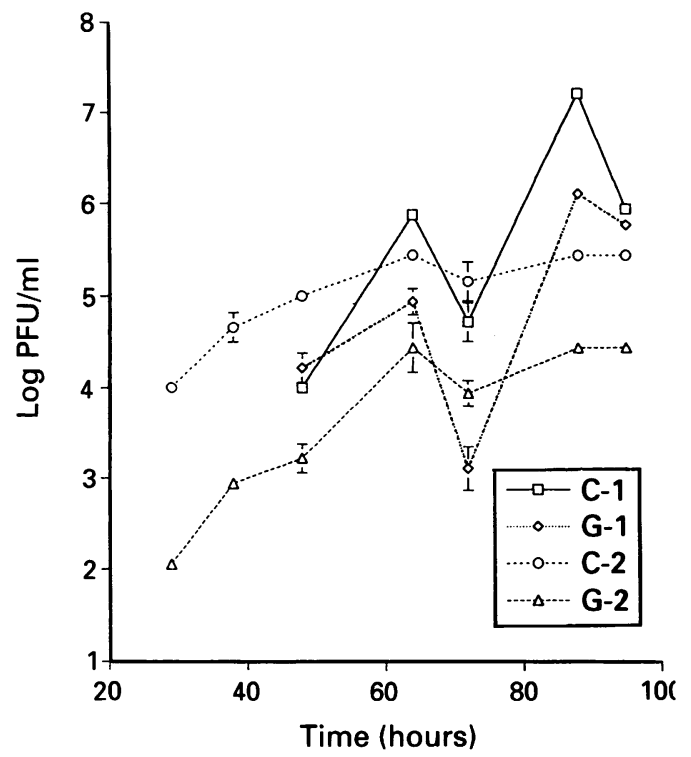

\section{Methods}

Material used for the culture of keratinocytes was purchased from the following sources: minimum essential medium (powder) was obtained from Gibco Life Technologies Inc., Chagrin Falls, Ohio, USA; ammonium metavenadate, ammonium molybdate tetrahydrate, copper sulphate pentahydrate, nickel chloride hexahydrate, tin chloride dihydrate, and zinc sulphate were obtained from JT Baker Chemicals B.V., Deventer, The Netherlands. Manganese sulphate was obtained from Cica Kanto Chemical Co., Tokyo, Japan. Selenic acid was obtained from Aldrich Chemical Co., Milwalkee, Wisconsin, USA. Other components for tissue culture were all purchased from Sigma Chemical Co., St Louis, Missouri, USA. Keratinocytes were obtained from surgical specimens of normal gingiva, cervix, skin, , and foreskin (newborn babies).

Tissues were washed with disinfectant for 10 to 30 minutes and rinsed with $30 \mathrm{mM}$

Figure 2 Growth curves of $H S V-1$ and $H S V-2$ in cultured media of infected cervix and normal gingiva. Each data point represents mean (range) of duplicate experiments (bar not drawn when titre difference between duplicate is less than $0.08 \log$ pfulml). C: cervix; G: normal gingiva; 1: $H S V-1 ; 2: H S V-2$.

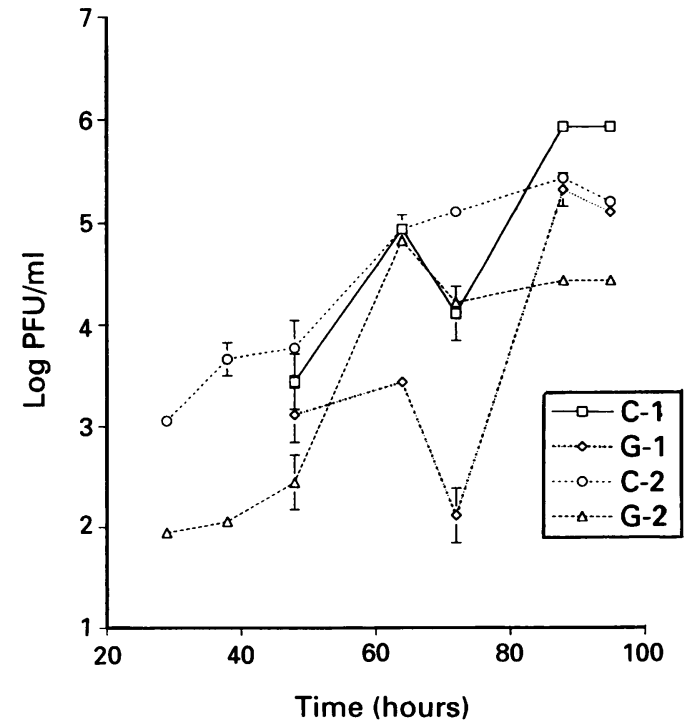

HEPES buffer (without $\mathrm{Ca}^{++}$and $\mathrm{Mg}^{++}$). The disinfectant contained $600 \mathrm{IU} / \mathrm{ml}$ penicillin, $0.2 \mathrm{mg} / \mathrm{ml}$ gentamycin, and $1 \%$ fungizone in $30 \mathrm{mM}$ HEPES buffer. After disinfection, unwanted portions of the tissue were removed, and the remainder minced into about $1 \mathrm{~mm}^{3}$ fragments, and three fragments were transferred, epidermis side up, to a sterile $35 \mathrm{~mm}$ Petri dish. When these fragments had adhered, minimum essential medium supplemented with $10 \%$ fetal calf serum was added. These cultures were then incubated in a humidified $5 \% \mathrm{CO}_{2}$ incubator at $35^{\circ} \mathrm{C}$.

When a substantial outgrowth of cells was observed, MCDB 153 serum free medium ${ }^{9} 10$ was used to substitute the serum containing medium. Cultures were re-fed twice weekly. Nearly confluent primary cultures were dissociated with $0.05 \%$ trypsin in $0.02 \%$ EDTA, pH $7 \cdot 45$, equilibrated with $10 \%$ glycerol in MCDB 153 serum free medium, and preserved in liquid nitrogen. The frozen keratinocytes were thawed and reconstituted in MCDB 153 serum free medium for further experiments. Cultures for our experiments were seeded with 100 to 200 cells per $\mathrm{cm}^{2}$.

The viruses were obtained from the poolwild-type clinical isolates. They were typed as HSV-1 and HSV-2 by direct immunofluorescence assay (Imagen UK). The viruses were propagated on the human embryonic lung cell (HEL), with less than three passages. The titre of each isolate was assayed and adjusted to about $10^{10}$ plaque forming units (pfu) $/ \mathrm{ml}$ for HSV-1 and about $10^{7} \mathrm{pfu} / \mathrm{ml}$ for $\mathrm{HSV}-2$ and then pooled. The viral infectivity from the pool was $8.7 \times 10^{9} \mathrm{pfu} / \mathrm{ml}$ of $\mathrm{HSV}-1$ and $1.38 \times 10^{7} \mathrm{pfu} / \mathrm{ml}$ of HSV-2.

Cultures of keratinocytes were infected with $10^{-1} \mathrm{pfu} / \mathrm{cell}$ of HSV-1 and HSV-2, respectively, and grown in MCDB 153 serum free medium. Culture fluid was removed at the intervals at which the titrations were done. The infected cell sheet was washed with 30 mM HEPES buffer and 1.5 ml MCDB 153 serum free medium was added. The combined medium and cells were frozen and thawed twice and titrated. Each experiment was performed in duplicate.

Cultures of keratinocytes were infected with $10^{-1} \mathrm{pfu} / \mathrm{cell}$ of HSV-1 and HSV-2, respectively. These cultures were fixed and stained at intervals. The fixative was $95 \%$ alcohol and the stain was a modified Papanicolaou technique. ${ }^{11}$

\section{Results}

INFECTIVITY IN CULTURED KERATINOCYTES

The infective titres of HSV-1 and HSV-2 obtained from infected cervix were higher than those obtained from infected normal gingiva. However, the pattern of the viral growth curve produced by HSV-1 was quite different from that produced by HSV-2 in both infected normal gingiva and infected cervix. But, for both HSV-1 and HSV-2, the viral growth pattern in normal gingiva was comparable with that in cervix. 


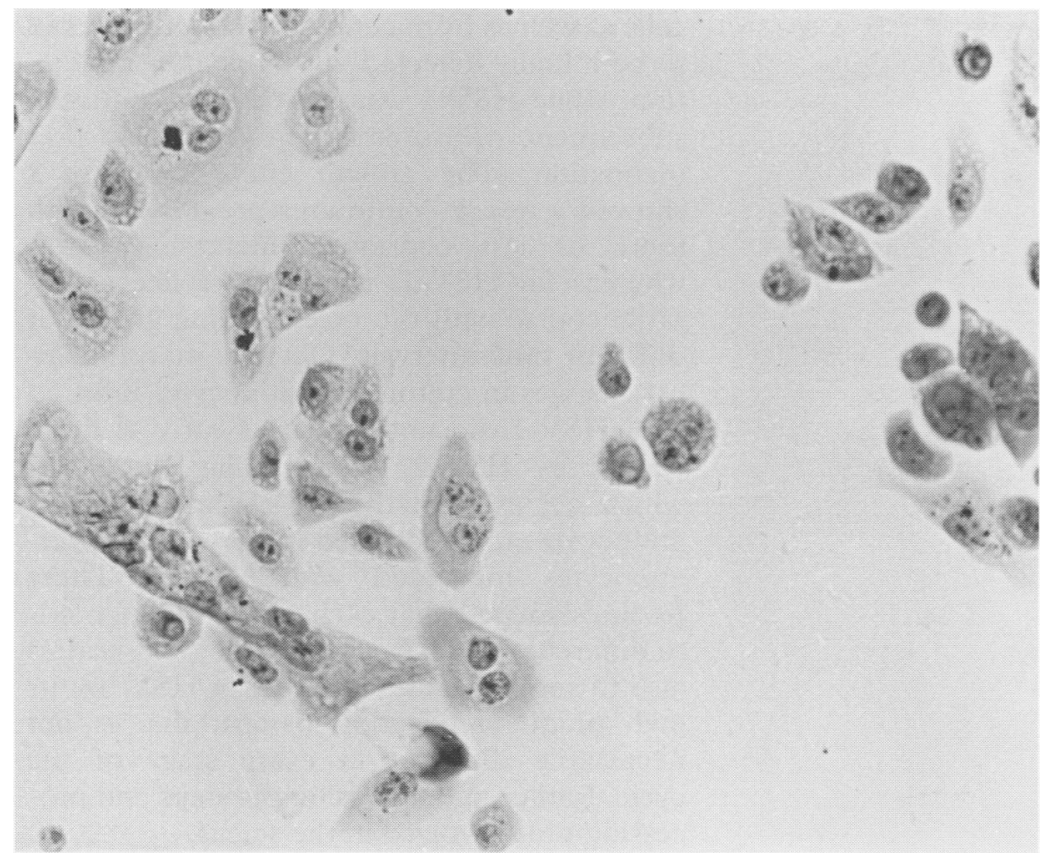

Figure 3 Cytopathic effects in normal gingiva, 67 hours after HSV-2 infection.

Figures 1 and 2 show the intracellular replication rate of HSV-1 in both cultured normal gingiva and cervix. There were substantial fluctuations during the different harvest time intervals. The intracellular viral infective titre was not detectable 38 hours after infection but then increased distinctly at both 48 hours and 64 hours after infection. At 72 hours the titre was substantially decreased and then increased again, with the highest titre at 88 hours after infection. At the end of the experimental period (95 hours after infection), the titre had fallen substantially for cervix; the titre for normal gingiva had fallen only slightly. The infective titre of the intrakeratinocyte viral component of $\mathrm{HSV}-2$, on the other hand, showed a steady increase 29 hours after infection to the end of the experimental period except that at 72 hours there was a decrease to $10^{5.11} \mathrm{pfu} / \mathrm{ml}$ for cervix and to $10^{3.94} \mathrm{pfu} / \mathrm{ml}$ for normal gingiva.

The infective titres obtained with the conditioned media for both cervix and normal

Cytopathic effects of cultured human keratinocytes from various anatomic sites infected by two HSV types and serial time intervals

\begin{tabular}{|c|c|c|c|c|}
\hline \multirow{2}{*}{$\begin{array}{l}\text { Time after infection } \\
\text { (hours) }\end{array}$} & \multicolumn{4}{|c|}{ Cytopathic effects in: } \\
\hline & Cervix & Normal gingiva & Trunk skin & Newborn foreskin \\
\hline \multicolumn{5}{|l|}{ HSV-1 } \\
\hline 24 & - & - & - & - \\
\hline 48 & \pm & \pm & \pm & \pm \\
\hline 72 & ++ & ++ & + & + \\
\hline 96 & $+++t$ & +++ & +++ & +++ \\
\hline 120 & ++++ & ++++ & ++++ & ++++ \\
\hline \multicolumn{5}{|l|}{ HSV-2 } \\
\hline 24 & \pm & - & - & - \\
\hline 48 & ++ & + & + & + \\
\hline 72 & ++++ & $++t$ & ++ & ++ \\
\hline 96 & ++++ & ++++ & ++++ & ++++ \\
\hline
\end{tabular}

$+=25 \%$ of cell population showing cytopathic change; $++=50 \%$ of cell population showing cytopathic change; $+++=75 \%$ of cell population showing cytopathic change; $++++=$ $100 \%$ of cell population showing cytopathic change. gingiva infection by HSV-1 and HSV-2 were similar for those of intracellular origin. Moreover, regardless of viral type, the titres with the conditioned media for cervix were higher than those with the conditioned media for normal gingiva. HSV-2 produced more constant infective titres in both cervix and normal gingiva in conditioned media. In HSV-2 infected cervix in conditioned media the infective titre increased following an almost parabolic line, with the highest titre at 88 hours. Correspondingly, in HSV-2 infected normal gingiva in conditioned media, the titre increased slightly between 29 and 48 hours after infection, then more rapidly between 48 and 64 hours. The titre then decreased at 72 hours followed by a further slight increase until the end of the experimental period. The infective titre for $\mathrm{HSV}-1$ from both infected cervix and normal gingiva in conditioned media showed a fluctuation pattern similar to that of the intracellular viral growth curves. The infective titres started to be detectable 48 hours after infection and continued to increase until 64 hours, decreased at 72 hours, and then increased thereafter to the end of the experiment.

\section{CYTOPATHIC EFFECTS IN CULTURED}

\section{KERATINOCYTES}

Cytopathic effects induced by a given viral type showed few distinguishable features whether in cultured normal gingiva, cervix, trunk skin, or in newborn foreskin. But some morphological changes and the time of appearance of cytopathic effects in the cultured keratinocytes caused by HSV-1 infection differed from those caused by HSV-2 infection. The cultured keratinocytes infected by type 2 viruses started to show cytopathic changes at 48 hours of incubation, becoming swollen with some even showing a tendency to fuse to each other, resulting in the formation of multinucleated giant cells. Ballooning degeneration with inconspicuous inclusion of nuclei and occasional vacuolation of cytoplasm were also noticed (fig 3). These cytopathic alterations initially involved patches of the subconfluent cultured cells and eventually affected the entire cell culture as time progressed, and the longer the incubation of the infected cells, the more discernible were the cytopathic changes. At 96 hours after infection, all HSV-2 infected cultured cells had shrunk and were globular in appearance. By contrast, the cytopathic effects in the cultured keratinocytes infected by HSV-1 took about 24 more hours of incubation to appear than those infected by HSV-2 (table). The cultured keratinocytes infected by HSV-1 revealed equivocal cytopathic changes at 48 hours of incubation and at 72 hours after infection patches of the HSV-1 infected cultured cells began to display remarkable cytopathic changes. In addition to the cytopathic effects similar to those described above, many of the HSV-1 infected cells were elongated and slender with bipolar cytoplasm (fig 4), and eventually all keratinocytes became globular in shape. 


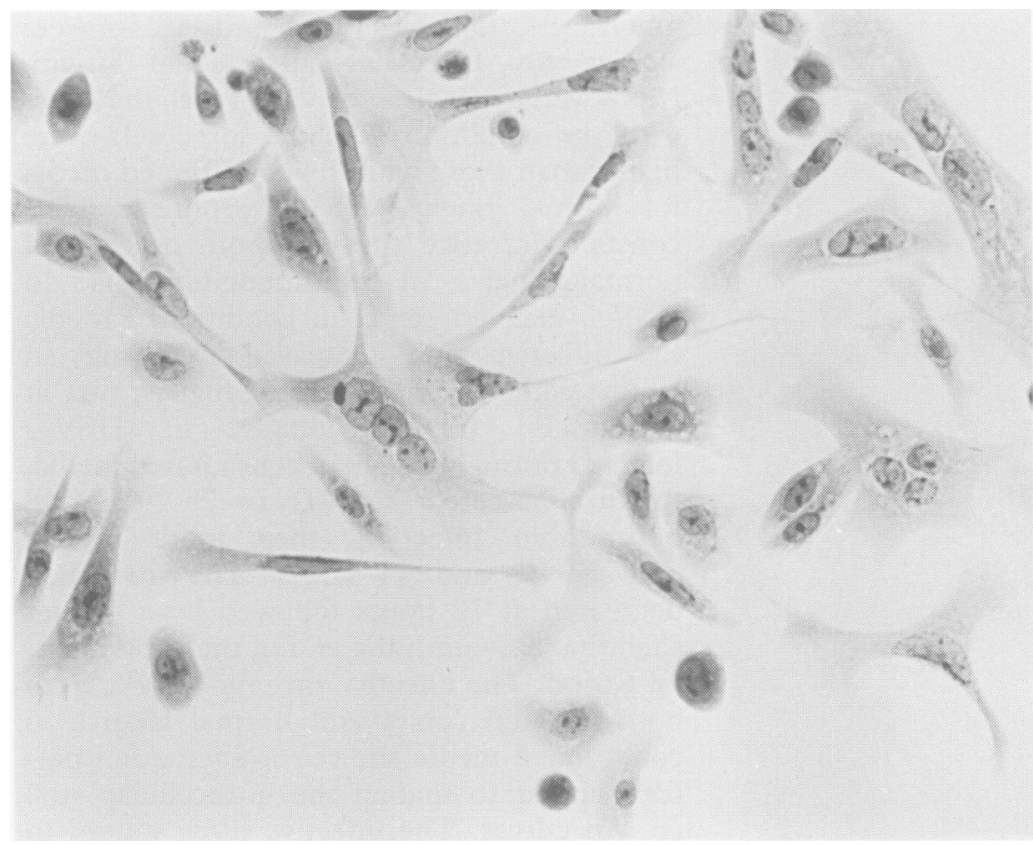

Figure 4 Cytopathic effects in normal gingiva, 67 hours after HSV-1 infection.

\section{Discussion}

Although the keratinocytes are the primary cells involved in clinical lesions caused by HSV, and normal human keratinocytes have been successfully grown in serum free defined culture medium over the past decade, ${ }^{912-14}$ studies of the progression of the cellular effects of HSV infection and the patterns of viral growth in cultured normal human keratinocytes have rarely been published. In this experiment we grew normal human keratinocytes from various anatomical sites in serum free defined culture medium MCDB 153. Both the progressive microscopic cellular changes and viral proliferation in cultured normal human keratinocytes infected by two subtypes of HSV were studied.

The results of our study clearly show that the reproductive cycles of HSV-1 and HSV-2 differ from each other in cultured normal human keratinocytes, irrespective of the anatomical source of the cultured cells. This is evident by their dissimilar growth curves and cytopathic effects on cultured keratinocytes. The HSV samples used in the present study were pooled and did not belong to any specific strains. This suggests that the differences in biological behaviour found between HSV-1 and HSV-2 were mainly due to type specificity and not intratypic variation. The use of a "pool" of virus certainly has its advantages. As very minor genotypic differences can result in substantial changes in biological characteristics, pooling of isolates lessens the chance of identifying only minor genotypic change and increases the chance of identifying true differences in growth characteristics in these two viral types. However, there is a theoretical disadvantage to using a "pool" of virus. It will not detect the differential growth of the constituent strains which occurs in culture. Infective HSV-2, measured by plaque titration from infected cultured cells as well as from conditioned media, began to be initially detected 29 hours after incubation, while HSV-1 was not detected until the subsequent measurement at 48 hours after incubation. The growth curve for HSV-2 showed a nearly continuous progression with more or less constantly increasing titres, whereas for HSV-1, it was more like a pulsatile course with the titres varying greatly at different time intervals. Obvious morphological changes in cultured keratinocytes infected with HSV-1 did not appear as early as those caused by HSV-2. The cellular elongation which occurred in the HSV-1 infected keratinocytes rarely occurred in the cultured keratinocytes incubated with HSV-2. These findings suggest that each type of $\mathrm{HSV}$ binds to different cell surface receptors ${ }^{15}$ and lead us also to suppose that each type of HSV has its own unique replication pattern that is not necessarily different at every stage of the cycle. Rather, it depends on the steps and processing time involved in the stages.

The infective titres of both HSV-1 and HSV-2 harvested from conditioned culture fluid and cells were higher in infected cultured cervix than in infected cultured normal gingiva. This phenomenon, coupled with the differences in viral growth curves between the two viral types, agrees with what Lafferty et al found in their clinical study concerning the subsequent recurring patterns of HSV infections affecting the same anatomical sites between type 1 and type $2,{ }^{1}$ and also with the recurrence rates between the oral-labial and genital regions infected either with types 1 or 2 virus. The data on infective viral titres obtained from co-incubated cultured human keratinocytes do not satisfactorily explain why HSV-1 is still more commonly associated with oral infection and HSV-2 is found mainly in genital regions.

The results from our study have disclosed the structural alterations in cultured normal human keratinocytes infected with HSV-1 and HSV-2 which manifest somewhat differently in the early phase of the cycle. However, the mature cytopathic changes in the late stages were almost the same and it was difficult to determine whether these changes were caused by HSV-1 or by HSV-2. This phenomenon correlates closely with the fact that the cytopathic changes in cellular samples collected from clinical samples cannot differentiate type 1 from type 2 virus. This correlation is easy to understand, because by the time the cellular samples were obtained from the clinical patients, the lesions usually had already existed for a period of time (roughly about a week) and were almost mature, and so the cytopathic changes in cellular samples from such lesions are supposed to be in the late stages of the cycle. The distinguishing manifestations of cytopathic effects in cultured keratinocytes between two individual related types of virus in the early stages of the cycle can be expected to be applied to the clinical laboratory in the near future, when culture of normal human keratinocytes is widely used, so that indications of the different lesions 
caused by those two types of virus could be obtained simply by the appearance of the cytopathic effects they produce.

1 Lafferty W, Remington M, Winter C, Fahnlander A Corey L. Natural history of concomitant pharyngeal and genital HSV infection: influence of viral type and anatomic site on recurrence rates [abstract]. Clin Res 1985;33:408A.

2 Corey L, Adams HG, Brown ZA, Holmes KK. Genital herpes simplex virus infection: clinical manifestations, course and complications. Ann Intern Med 1983;98: 958-72.

3 Reeves WC, Corey L, Adams HG, Vontver LA, Holmes KK. Risk of recurrence after first episodes of genital herpes: relation to HSV type and antibody response. $N$ Engl pes: relation to HSV type an

4 Lafferty WE, Coombs RW, Benedetti J, Critchlow C, Corey L. Recurrences after oral and genital herpes simplex virus infection: influence of site of infection and viral type. $N$ Engl $\mathcal{F}$ Med 1987;316:1444-9.

5 Birch J, Fink CG, Skinner RB, Thomas GH, Jordan JA. Replication of type 2 herpes simplex virus in human endocervical tissue in organ culture. Br $\mathcal{f}$ Exp Pathol 1976;57:460-71.

6 Plummer G, Waner JL, Bowling CP. Comparative studies of type 1 and type 2 herpes simplex virus. $B r \mathcal{F} \operatorname{Exp}$ Pathol 1968;49:202-8.
7 Teplitz RL, Valco Z, Rundall T. Comparative sequential cytologic changes following in vitro infection with herpes types 1 and 2. Acta Cytol 1971;15:455-9.

8 Norskov-Lauristen N, Aboagye-Mathisen G, Juhl CB, Petersen PM, Zachar V, Ebbesen P. Herpes simplex virus infection of cultured human term trophoblasts. $\mathcal{7 M e d}$ Virol 1992;36:162-6.

9 Boyce ST, Ham RG. Calcium-regulated differentiation of normal human epidermal keratinocytes in chemically normal human epidermal keratinocytes in chemically defined clonal culture and serum free

F Invest Dermatol 1983;80(Suppl):33-40.
10 Pirisi L, Yasumoto S, Feller M, Doniger J, DiPaolo JA. Transformation of human fibroblasts and keratinocytes with human papilloma virus type 16 DNA. $\mathcal{f}$ Virol 1987 ; 61:1061-6.

11 Cuyler WK. The recognition of carcinoma-in-situ of the cervix by Papanicolaou's method. In: Progress in fundamental medicine. Philadelphia: Lee \& Febiger, 1952: 261-76.

12 Lin HY. Production of collagenase by human keratinocytes. [Thesis]. University of Alabama at Birmingham, 1987:1-72.

13 Wille I Jr, Pittelkow MR, Shipley GD, Scott RE. Integrated control of growth and differentiation of normal human prokeratinocytes cultured in serum-free mal human prokeratinocytes cultured in serum-free medium: clonal analysis, growth kinetics

14 Schafer IA, Kovach M, Price RL, Fratianne RB. Human keratinocytes cultured on collagen gels from an epidermis which synthesizes bullous pemphigoid antigens and $\alpha 2 \beta 1$ integrins and secretes laminin, type IV collagen and heparan sulfate proteoglycan at the basal cell surface. Exp Cell Res 1991;195:443-57.

15 Corey L, Spear G. Infections with herpes simplex virus. N Engl f Med 1986;314:686-91. 